\title{
An interesting case of ocular involvement in a case of dystrophic epidermolysis bullosa with conjunctival blistering without eyelid or corneal disease; a case
} report

\begin{abstract}
Epidermolysis bullosa (EB) is an autoimmune mucocutaneous disorder associated with abnormalities of the basement membrane zone of skin and mucous membranes. It is characterized by blister formation in response to minor trauma to the skin and mucous membranes. Here, we present a case of dystrophic epidermolysis bullosa (DEB) in 4years old boy with conjunctival blister formation without eyelid or corneal involvement. He was presented with redness in right eye and active and chronic blistering skin lesions on the elbows, fingers, knees, legs, toes and back of the pinna with scarring in some areas. He had dystrophy of nails and absence of nails in some toes. The oral mucosa was normal and the patient did not have any swallowing complains or other gastrointestinal disorders. A small blister formation was noted in the superior temporal quadrant of conjunctiva in right eye with episcleral congestion. He was diagnosed as a case of autosomal dominant variant of dystrophic epidermolysis bullosa (DDEB), confirmed with biopsy and genetic analysis. So, ophthalmic complications are common in DDEB, but the involvement of ocular structures varies widely among different patients and different subtypes of the disease. Conjunctival blistering without eyelid or corneal disease is one of the most common ophthalmic complications. Protection of the eye from minor trauma such as rubbing may help prevent ocular complications. The major treatment modality is the use of ocular lubricants.
\end{abstract}

Keywords: dystrophic epidermolysis bullosa, autoimmune, mucocutaneous, conjunctival blister, blistering skin lesions
Volume 9 Issue I - 2019

\author{
Das Mohapatra Shyam Sundar,' Das \\ Dipankar, ${ }^{2}$ Bhattacharjee Harsha, ${ }^{3}$ Agarwal \\ Shashank' \\ 'DNB Ophthalmology Resident, Sri Sankaradeva Nethralaya, \\ India \\ ${ }^{2}$ Department of Ocular Pathology, Uveitis and \\ Neuroophthalmology Services, India \\ ${ }^{3}$ Medical Director, Sri Sankaradeva Nethralaya, India
}

Correspondence: Dr. Shyam Sundar Das Mohapatra, Sri Sankaradeva Nethralaya, 96, Basistha Road, Guwahati, Assam, India, Pin - 78I028, Tel +9| 9239576199,

Emaildrssdasmohapatra@gmail.com

Received: January 17, 2019 | Published: February 18, 2019

\section{Introduction}

Epidermolysis bullosa (EB), an autoimmune mucocutaneous disorder, ${ }^{1}$ associated with abnormalities of the basement membrane zone of skin and mucous membranes, ${ }^{2}$ is characterized by blister formation in response to minor trauma to the skin and mucous membranes, ${ }^{1}$ which started during infancy. ${ }^{2}$ Most frequently, this disorder presents in hereditary forms, but an acquired form also exists. ${ }^{2}$ The hereditary forms have several subtypes, including epidermolysis bullosa simplex (autosomal dominant), junctional epidermolysis bullosa (autosomal dominant) and dystrophic epidermolysis bullosa (DEB, autosomal dominant or recessive). ${ }^{1}$ In the DEB subtype, a sub epidermal blister beneath the basal lamina is a characteristic feature. ${ }^{3}$ Now, many of the subtypes of EB have been characterised by mutations of various genes affecting different elements of the basement membrane zone. Genetic studies have found mutations in the COL7A1 gene in DEB (both dominant and recessive types). This gene is responsible for collagen type VII synthesis, which acts as the coordinating agent for tissue collagen fibrils. So, due to these mutations, collagen fibrils are get disrupted. ${ }^{4}$ The clinical manifestations and the severity varies thoroughly among dominant and recessive forms of DEB. The dominant form of DEB (DDEB) usually present soon after birth or during early infancy or early childhood with skin blistering and dermal scarring. Dystrophic nails are also a common presentation in the dominant variety, ranges from alteration in the shape of the nail to complete absence of nail. ${ }^{1}$ Recessive type of DEB (RDEB) is commonly manifests at birth with more severe clinical manifestations as compared to the dominant form with extensive dermal blister formation over the extremities, knees and elbows result in joint deformity and polysyndactyly. Mucocutaneous scars are common in the recessive form in comparison with dominant form, which lead to development of microstomia and ankyloglossia, can also leads to development of squamous cell carcinoma. Gastrointestinal strictures can also occur and cause problems with eating and swallowing. ${ }^{5}$ Ocular manifestations of EB varies widely, including conjunctival blister formation, corneal ulceration, symblepharon formation, blepharitis, ectropion, nasolacrimal duct obstruction, disorder of vision and even blindness in some cases. ${ }^{6}$ Herein, we report a case of DDEB who developed conjunctival blister formation without any eyelid or corneal disease.

\section{Case report}

A 4years old male patient was reported to our hospital with complains of redness in right eye and itching and discharge in both eyes. He had history of blistering skin lesions on the extremities since the age of three months. He was diagnosed as a case of DEB by dermatologist, proven by biopsy. On meticulous history taking and general examination, he was found to be a case of autosomal dominant variant of DEB (DDEB), which was confirmed by genetic analysis. On general examination, he had active and chronic blistering 
skin lesions on the elbows, fingers, knees, legs, toes (Figure 1) and back of the pinna (Figure 2), which had led to scarring in some areas. He had dystrophy of nails and absence of nails in some toes (Figure 3). The oral mucosa was normal and the patient din not have any swallowing complains or other gastrointestinal disorder with abdominal ultrasonography finding within normal limit. On ophthalmic examination, there was a small blister formation in the superior temporal quadrant of conjunctiva in right eye with episcleral congestion (Figure 4). The rest of ophthalmic examination was unremarkable. On investigation, irregular astigmatism was found in both eyes on corneal topography. He was prescribed lubricating eye drops for application in both the eyes and advised for regular followup. The parents were counselled to prevent the child from rubbing of eyes and protect the exposed areas of the skin even from minor trauma to prevent development of new skin lesions.

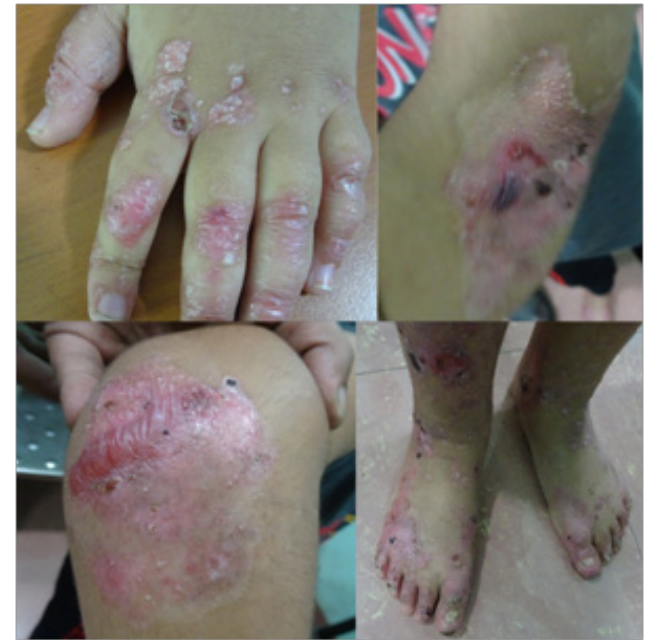

Figure I Shows active and chronic blistering skin lesions on the elbows, fingers, knees, legs, toes with scarring in some areas.

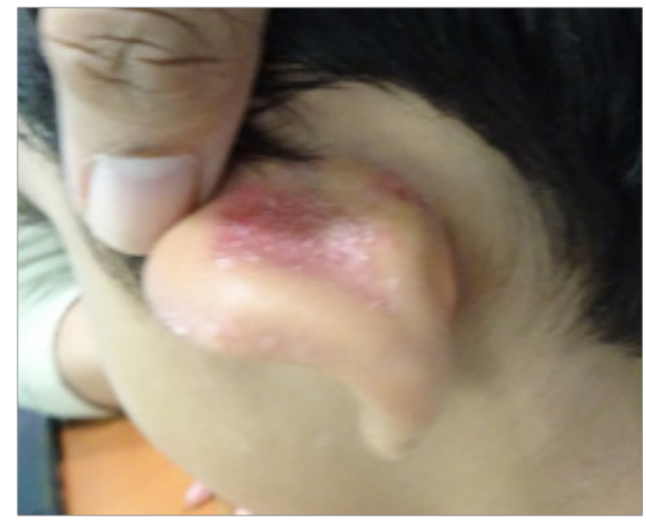

Figure 2 Shows active and chronic blistering skin lesions on the back of the pinna.

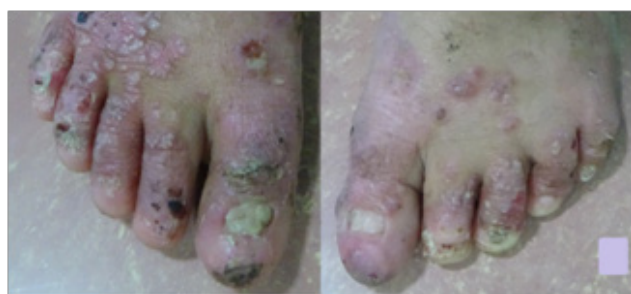

Figure 3 Shows dystrophy of nails and absence of nails in some toes.

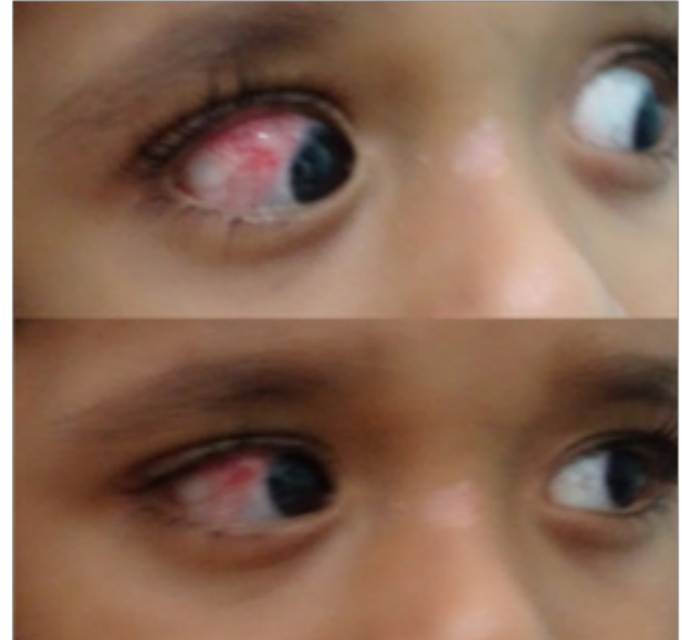

Figure 4 Shows small blister formation in the superior temporal quadrant of conjunctiva in right eye with episcleral congestion.

\section{Discussion}

Dystrophic epidermolysis bullosa is a skin disorder resulting in dissociation of its superficial layers. This disorder is the most severe type of epidermolysis bullosa. ${ }^{5}$ Tong et al. $^{2}$ reported that Autosomal dominant dystrophic EB $(n=28)$ : only one patient $(4 \%)$ had significant ocular involvement consisting of conjunctival blistering without eyelid or corneal disease. Previously McDonnell ${ }^{7}$ studied a series of 11 patients with dystrophic EB and reported $73 \%$ of patients were noted to have eye changes. Lin AN et al. ${ }^{8}$ reported in the review of ophthalmic findings in 204 patients with epidermolysis bullosa that ocular complications were seen in $26 \%$ of the 204 patients. Abnormal structure of collagen type VII in patients suffering from DEB results in deficient tissue repair leading to scar and fibrous formation. ${ }^{4}$ The patient presented herein was a case of DEB who developed conjunctival blisters and episcleral congestion. The patient also had complain of itching and habit of rubbing of eyes which may cause recurrent lesion and with more severity and may lead to symblepharon formation. Use of protective glasses and avoiding minor trauma such as rubbing of eyes can be beneficial for patients with DEB to prevent ocular complications such as symblepharon formation. ${ }^{1}$

\section{Conclusion}

Ophthalmic complications are common in DDEB, but the incidence of involvement of ocular structures varies widely among different patients as well as among different subtypes. Conjunctival blistering without eyelid or corneal disease is one of the most common ophthalmic complication. Protection of the eye from minor trauma such as rubbing may help prevent ocular complications. The major treatment modality is the use of ocular lubricants.

\section{Funding details}

No funding has been received.

\section{Acknowledgments}

None.

\section{Conflicts of interest}

There is no conflicts of interest among the authors. 


\section{References}

1. Mahdavi M, Javadi MA. External Ocular Manifestations in Autosomal Dominant Dystrophic Epidermolysis Bullosa; a Case Report. $J$ Ophthalmic Vis Res. 2008;3(1):70-73.

2. Tong L, Hodgkins PR, Denyer Jet al. The eye in epidermolysis bullosa. $\mathrm{Br}$ J Ophthalmol. 1999;83(3):323-326.

3. Fine JD, Johnson LB, Weiner M, et al. Eye involvement in inherited epidermolysis bullosa: experience of the National Epidermolysis Bullosa Registry. Am J Ophthalmol. 2004;138(2):254-262.

4. Sawamura D, Nizeki H, Miyagawa S, et al. Novel indel COL7A1 mutation 8068 del17ins GA causes dominant dystrophic epidemolysis bullosa. Br J Dermatol. 2006;154(5):995-997.
5. Azrak B, Kaevel K, Hofmann L, et al. Dystrophic epidermolysis bullosa: oral findings and problems. Spec Care Dentist. 2006;26(3):111-115.

6. Matsumoto Y, Dogru M, Tsubota K. Ocular surface findings in HallepauSiemens subtype of dystrophic epidemolysis bullosa: report of a case and literature review. Cornea. 2005;24(4):474-479.

7. McDonnell PJ, Spalton DJ. The ocular signs and complications of epidermolysis bullosa. J R Soc Med. 1988;81(10):576-578.

8. Lin AN, Murphy F, Brodie SE, et al. Review of ophthalmic findings in 204 patients with epidermolysis bullosa. Am J Ophthalmol. 19941;118(3):384-390. 